\title{
Modified Polyaniline Nanofibres for Ascorbic Acid Detection
}

\author{
Larisa Florea $^{1}$, Emer Lahiff ${ }^{1}$ and Dermot Diamond ${ }^{1}$ \\ ${ }^{1}$ CLARITY: The Centre for Sensor Web Technologies, Dublin City University, Dublin 9, \\ Ireland.
}

\begin{abstract}
Polyaniline nanofibres (PAni) can be surface modified to improve electroactivity over a broader $\mathrm{pH}$ range. The technique we describe here can be used to attach carboxylic acid terminated substituents. Modified nanofibres maintain their high surface area, and ability to switch between different redox states. These properties make the material suitable for sensing applications. Unlike unmodified PAni, the functionalised material is self-doping and hence more stable in higher $\mathrm{pH}$ solutions. Here we demonstrate how modified PAni fibres can be used for the detection of ascorbic acid.
\end{abstract}

\section{INTRODUCTION}

Ascorbic acid (AA) is an effective reducing agent, and a powerful antioxidant in food. Various methods have been reported for the determination of ascorbic acid, such as spectrophotometry [1], fluorimetry [2], chromatography [3] and electrochemical detection [4]. Modified electrodes have been applied for the determination of AA electrochemically $[5,6]$, and using conducting polymer electrodes specifically has advantages such as three-dimensional mediator distribution, broad potential window and good electrode stability [7-9].

Polyaniline (PAni) is an example of a stable conducting polymer whose properties (optical, electrical and electrochemical) change in response to the immediate molecular environment of the material. PAni can be cheaply and conveniently synthesized, and thus has huge potential for sensor applications. The nanofibre form of PAni can be synthesised directly by chemical means, such as interfacial polymerisation. Synthesis by this method is scalable, whereby increasing the amount of reactant results in a greater yield of the nanofibre product. By using PAni nanofibres (versus bulk) the surface area exposed to the target molecules can be dramatically increased, resulting in a sensor material with enhanced sensitivity and improved response times [10-14]. Our focus in this paper is on the covalent modification of these nanostructures post-polymerisation [15]. We functionalise the nanofibres with carboxylic acid terminated side-chains, resulting in self-doped PAni. Using the technique described, control over the extent of functionalisation can be achieved [16] and, the modified fibres can be used for the successful detection of ascorbic acid.

\section{EXPERIMENT}

Aniline (BDH), $\mathrm{HCl}$ (Fisher Scientific), ammonium peroxydisulfate (Aldrich), ascorbic acid (Aldrich), mercaptopropanoic acid (Aldrich), mercaptohexadecanoic acid (Aldrich) and 
Phosphate Buffered Saline (Aldrich) were used. The aniline monomer was purified by vacuum distillation before use. Other chemicals were used as received.

Polyaniline nanofibres were synthesised by interfacial polymerisation between an aqueous and an organic layer, as reported previously [10]. The product was purified by centrifugation ( $4000 \mathrm{rpm} / 5 \mathrm{~min} / 3$ cycles) and suspended as a colloid in deionised water. Fibres were then modified with an acid-terminated thiol by refluxing at $100{ }^{\circ} \mathrm{C}$ for 2 hours in an aqueous buffer at $\mathrm{pH} 4$ [17]. The product was purified by centrifugation $(4000 \mathrm{rpm} / 5 \mathrm{~min} / 3$ cycles) and re-suspended as a colloid in deionised water.

Electrochemistry of the fibres was investigated using a $\mathrm{CH}$ Instruments Electrochemical Analyser. Cyclic voltammetry results were obtained using phosphate buffered saline solution (PBS, $\mathrm{pH}=7.4$ ) as the electrolyte at a scan rate of $10 \mathrm{mV} / \mathrm{s}$. The test solutions were deaerated by passing a stream of pure nitrogen for at least $20 \mathrm{~min}$ prior to experiments, and all experiments were carried out at room temperature around $20{ }^{\circ} \mathrm{C}$. A glassy carbon working electrode, a Pt wire counter-electrode and a $\mathrm{Ag} / \mathrm{AgCl}$ reference electrode were used. Samples were drop cast onto a polished glassy carbon electrode, to form insoluble coatings after drying in air for several hours.

Modified nanofibres were imaged using field emission scanning electron microscopy (FESEM) at an accelerating voltage of $20 \mathrm{kV}$ on a S-4300 Hitachi system. UV-vis spectroscopy was carried out on a Perkin Elmer UV-vis NIR Lambda 900 Spectrometer at $1 \mathrm{~nm}$ resolution.

\section{DISCUSSION}

Polyaniline nanofibres were modified by a simple reflux process to attach carboxylic acid terminated thiol side-chains (PAni-COOH). The covalent attachment was verified using FTIR and NMR as previously published by the authors [16]. Post-modification nanofibres maintain their nanostructure, and hence high surface area (Figure 1).
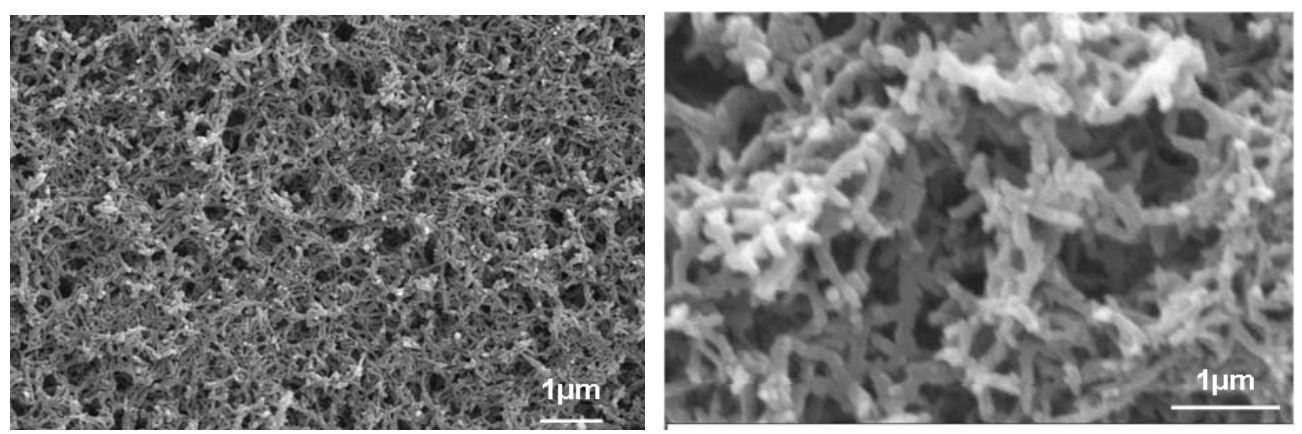

Figure 1: FESEM images of PAni nanofibres (left), and PAni-COOH nanofibres (right). Images confirm that the PAni maintains its nanostructured form post-functionalisation.

The modified polyaniline nanofibres maintain their ability to switch between different redox states, making them suitable for sensing applications. Cyclic voltammetry of PAni-COOH nanofibres is similar to that of PAni, under acidic conditions. Redox cycling for both PAni and $\mathrm{PAni}-\mathrm{COOH}$ is reversible, and current flow for both oxidation and reduction is approximately equal (Figure 2). 


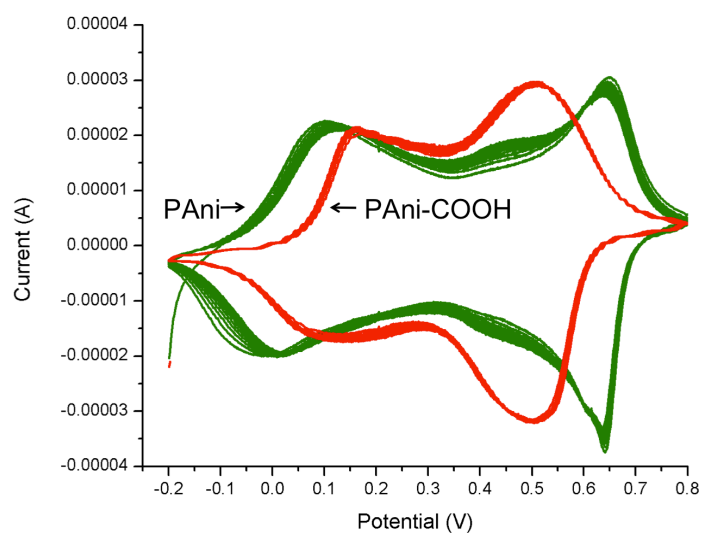

Figure 2: Cyclicvoltammetry of PAni nanofibres was compared with that for the functionalised material $(\mathrm{pH}=1)$.

Modification causes a slight change in the electrochemical profile, however PAni$\mathrm{COOH}$ remains clearly redox active.

Polyaniline nanofibres are of interest for sensing applications as they adapt their redox state, and chemical structure, depending on the local environment. The UV-Vis spectra of doped polyaniline (known as the emeraldine salt form), shows three characteristic absorption bands at $350 \mathrm{~nm}, 420 \mathrm{~nm}$ and $770 \mathrm{~nm}$. These bands correspond to $\pi-\pi^{*}$ band-gap absorption, low wavelength polaron- $\pi^{*}$ band and high wavelength $\pi$-polaron band, respectively. The undoped form of polyaniline (known as the emeraldine base form), shows a low wavelength $\pi-\pi^{*}$ band at $320 \mathrm{~nm}$ and a strong absorption band at $575 \mathrm{~nm}$. These are attributed to local charge transfer between a quinoid ring and the adjacent imine-phenyl-amine unit [18].

PAni at $\mathrm{pH}>4$ has the emeraldine base form, which does not have the polaron transition (due to low concentration of acid, $\mathrm{H}^{+}$). In the $\mathrm{UV}-\mathrm{V}$ is absorption spectra of carboxylated polyaniline (PAni-COOH), the generation of the polaron was observed, even with this system at $\mathrm{pH} 7.4$ (Figure 3). Therefore, UV-vis spectra demonstrate that the PAni-COOH is self-doped in media of $\mathrm{pH}>4$. The intensity of the polaron transition band is greater when the mole ratio between the monomer and carboxylic acid is increased. This self-doping polyaniline shows better dispersability and stability (in PBS), over time, when compared with unmodified polyaniline.

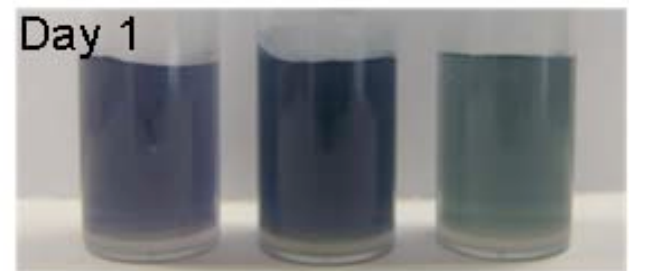

Day 3
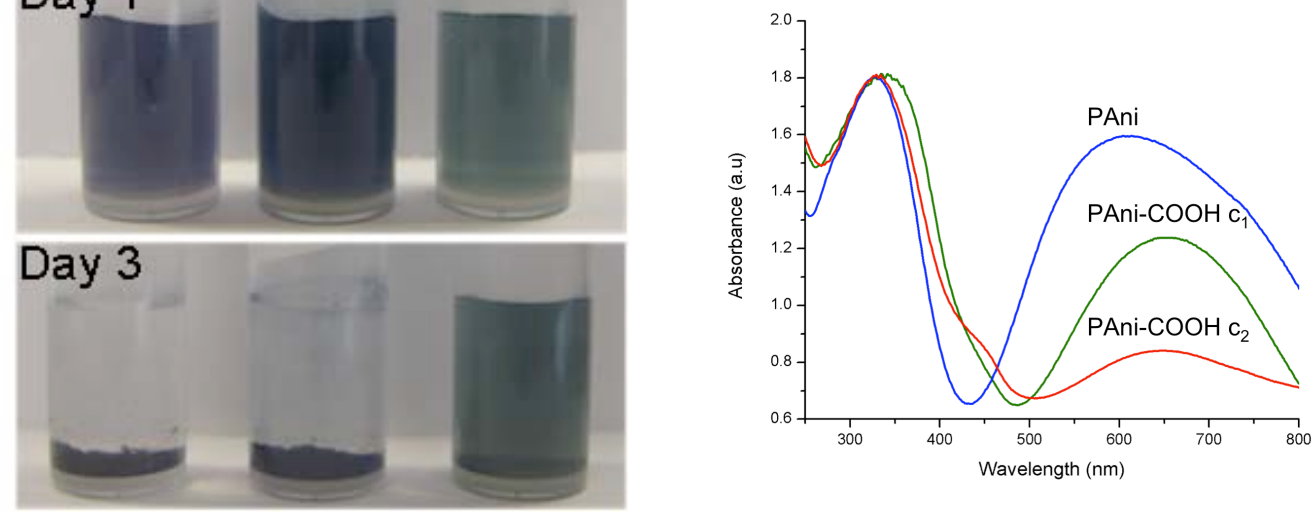

Figure 3: Pictures of PAni dispersion (at $\mathrm{pH}$ 7.4), on day 1 and day 3. Images left-to-right show unmodified PAni, PAni-COOH $\mathrm{c}_{1}$ (concentration 1), and PAni-COOH $\mathrm{c}_{2}$ (concentration 2), where $\mathrm{c}_{1}<\mathrm{c}_{2}$. UV-Vis Spectra of PAni and PAni-COOH (in PBS, $\mathrm{pH}$ 7.4), are shown on the far right. 


\section{Ascorbic acid detection using the PAni-COOH electrode}

As PAni-COOH is more stable than PAni in neutral $\mathrm{pH}$ environments, the material was examined as a simple biosensor. Here we demonstrate the material for use in electrochemical ascorbic acid (AA) detection. AA has an oxidation peak at $\sim 0.5 \mathrm{~V}$. Using the PAni- $\mathrm{COOH}$ modified electrode however, we can detect the AA at a much lower potential of $0.05 \mathrm{~V}$. This peak corresponds to the oxidation peak of PAni- $\mathrm{COOH}$, and it was observed to increase when AA was added (Figure 4). The magnitude of this peak continues to increase for greater concentrations of AA.

The reaction process can be described as: AA in solution diffused toward the polymer modified electrode surface and reacted with the oxidized state polyaniline which led to the increase of the reduced state polyaniline. This increasing of reduced state polyaniline resulted in the increase of oxidation currents [9]. CV curves by continuous cyclic sweep in AA solution did not bring about any change, which indicated that this polymer-modified electrode was not polluted by the adsorption of AA.

For higher concentrations of AA ( $>3 \mathrm{mmol} / \mathrm{l})$, a second redox peak appears at $\sim 0.3 \mathrm{~V}$. This is not a PAni redox peak and we suggest that it reflects a shifted AA peak. As the amount of AA increases, the position of this second redox peak shifts further towards the $0.5 \mathrm{~V}$ of AA. For example, at $10 \mathrm{mmol} / 1 \mathrm{AA}$ the peak is at $0.4 \mathrm{~V}$ (data not shown).

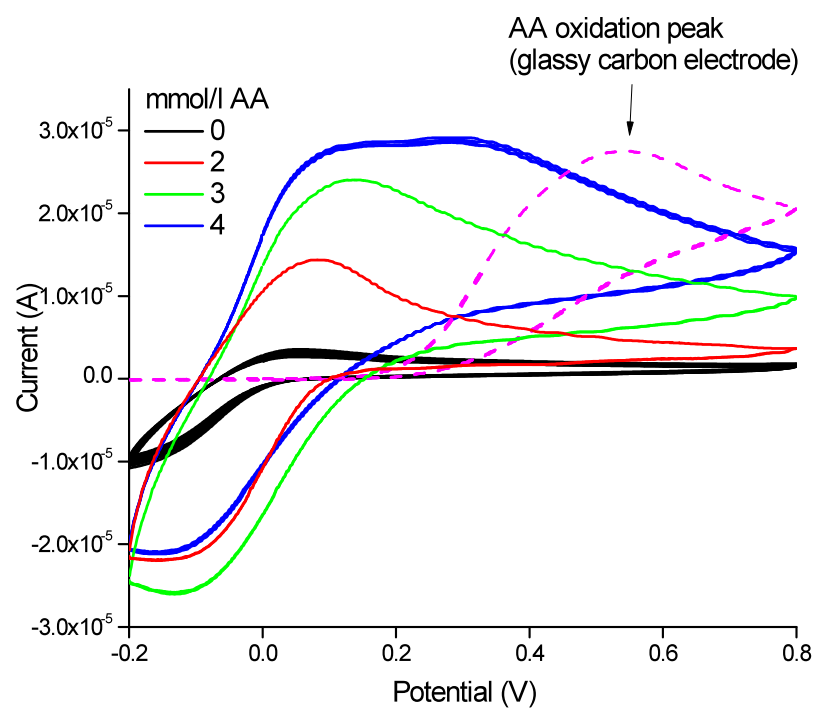

Figure 4: The CVs of a PAni-COOH modified electrode in the presence of $0,2,3$, and $4 \mathrm{mmol} / \mathrm{l}$ ascorbic acid. The CV obtained for a bare glassy carbon electrode in the presence of ascorbic acid is also shown for comparison.

\section{CONCLUSIONS}

One of the major limitations of PAni is that it loses the electroactivity at $\mathrm{pH}>4$. By functionalising PAni with carboxylic acid terminated side-chains we create a self-doping 
nanomaterial that presents good electroactivity over a wide $\mathrm{pH}$ range. Here we demonstrate that this new material exhibits good electrocatalytic activity for the oxidation of ascorbic acid. The carboxyl group-functionalised polyaniline offers new opportunities in the area of selfassembly, and chemical and biological sensors. As the carboxyl dopant group is a structural part of the main polymer chain it will not leach away, and can be further functionalised by other guest molecules via esterification. This polymer could be further applied for the construction of an enzyme biosensor, which would greatly widen the use of conductive polyaniline in biological electrochemistry.

\section{ACKNOWLEDGMENTS}

The authors acknowledge funding from Science Foundation Ireland under SFI 07/CE/I1 147 - "CLARITY: Centre for Sensor Web Technologies" and IRCSET - The Embark Initiative.

\section{REFERENCES}

1. O. W. Lau, S. F. Luk and K. S. Wong, Analyst 112 (7), 1023-1025 (1987).

2. $\quad$ N. Moll and J. P. Joly, Journal of Chromatography 405, 347-356 (1987).

3. H. P. Huang, R. X. Cai, Y. M. Du and Y. N. Zeng, Anal. Chim. Acta 309 (1-3), 271-275 (1995).

4. K. Matsumoto, J. J. B. Baeza and H. A. Mottola, Anal. Chem. 65 (13), 1658-1661 (1993).

5. J. Facci and R. W. Murray, Anal. Chem. 54 (4), 772-777 (1982).

6. A. P. Doherty, M. A. Stanley and J. G. Vos, Analyst 120 (9), 2371-2376 (1995).

7. A. M. Yu, D. M. Sun, H. Y. Gu and H. Y. Chen, Analytical Letters 29 (15), 2633-2643 (1996).

8. E. W. Kristensen, W. G. Kuhr and R. M. Wightman, Anal. Chem. 59 (14), 1752-1757 (1987).

9. J. J. Xu, D. M. Zhou and H. Y. Chen, Fresenius Journal of Analytical Chemistry 362 (2), 234-238 (1998).

10. J. X. Huang, S. Virji, B. H. Weiller and R. B. Kaner, Journal of the American Chemical Society 125 (2), 314-315 (2003).

11. S. Virji, R. B. Kaner and B. H. Weiller, Journal of Physical Chemistry B 110 (44), 22266-22270 (2006).

12. S. Virji, R. B. Kaner and B. H. Weiller, Chemistry of Materials 17 (5), 1256-1260 (2005).

13. S. Virji, J. X. Huang, R. B. Kaner and B. H. Weiller, Nano Letters 4 (3), 491-496 (2004).

14. S. Virji, J. D. Fowler, C. O. Baker, J. X. Huang, R. B. Kaner and B. H. Weiller, Small 1 (6), 624-627 (2005).

15. E. Lahiff, Bell, S, Diamond, D., Mater. Res. Soc. Symp. Proc. 1054, 1054-FF10051005. (2008).

16. E. Lahiff, T. Woods, W. Blau, G. G. Wallace and D. Diamond, Synthetic Metals 159 (78), 741-748 (2009).

17. E. Lahiff, Scarmagnani, S, Schazmann, B, Cafolla, A, Diamond, D., International Journal of Nanomanufacturing. 5 (1/2), 88-99 (2010). 
18. S. Atkinson, H. S. O. Chan, A. J. Neuendorf, S. C. Ng, T. T. Ong and D. J. Young, Chemistry Letters (3), 276-277 (2000). 